\title{
Mechanical Strength Improvements of Carbon Nanotube Threads through Epoxy Cross-Linking
}

\author{
Qingyue Yu ${ }^{1,2}$, Noe T. Alvarez ${ }^{3, *}$, Peter Miller ${ }^{3}$, Rachit Malik ${ }^{4}$, Mark R. Haase ${ }^{3}$, Mark Schulz ${ }^{4}$, \\ Vesselin Shanov ${ }^{3,4, *}$ and Xinbao $\mathrm{Zhu}^{1, *}$ \\ 1 Department of Chemical Engineering, Nanjing Forestry University, Nanjing 210037, China; \\ qingyueyunj@126.com \\ 2 Department of Chemical Engineering, Nanjing Polytechnic Institute, Nanjing 210048, China \\ 3 Department of Biomedical, Chemical and Environmental Engineering, University of Cincinnati, Cincinnati, \\ OH 45221, USA; millepj@mail.uc.edu (P.M.); haasemr@mail.uc.edu (M.R.H.) \\ 4 Department of Mechanical and Materials Engineering, University of Cincinnati, Cincinnati, OH 45221, USA; \\ malikrt@mail.uc.edu (R.M.); schulzmk@ucmail.uc.edu (M.S.) \\ * Correspondence: alvarene@ucmail.uc.edu (N.T.A.); shanovvn@ucmail.uc.edu (V.S.); \\ zhuxinao@njfu.com.cn (X.Z.); Tel.: +1-832-623-3451 (N.T.A.); +1-513-556-2461 (V.S.); +86-25-8542-7532 (X.Z.)
}

Academic Editor: Changle Chen

Received: 23 October 2015; Accepted: 18 January 2016; Published: 25 January 2016

\begin{abstract}
Individual Carbon Nanotubes (CNTs) have a great mechanical strength that needs to be transferred into macroscopic fiber assemblies. One approach to improve the mechanical strength of the CNT assemblies is by creating covalent bonding among their individual CNT building blocks. Chemical cross-linking of multiwall CNTs (MWCNTs) within the fiber has significantly improved the strength of MWCNT thread. Results reported in this work show that the cross-linked thread had a tensile strength six times greater than the strength of its control counterpart, a pristine MWCNT thread (1192 MPa and $194 \mathrm{MPa}$, respectively). Additionally, electrical conductivity changes were observed, revealing $2123.40 \mathrm{~S} \cdot \mathrm{cm}^{-1}$ for cross-linked thread, and $3984.26 \mathrm{~S} \cdot \mathrm{cm}^{-1}$ for pristine CNT thread. Characterization suggests that the obtained high tensile strength is due to the cross-linking reaction of amine groups from ethylenediamine plasma-functionalized CNT with the epoxy groups of the cross-linking agent, 4,4-methylenebis(N,N-diglycidylaniline).
\end{abstract}

Keywords: carbon nanotube thread; carbon nanotube fiber; high tensile strength; epoxy cross-linking; 4,4-methylenebis (N,N-diglycidylaniline)

\section{Introduction}

Vertically-aligned carbon nanotubes (CNTs) are one-dimensional nanomaterials with extremely high tensile strength [1,2], elastic modulus [1,3], large aspect ratio, low density, good chemical and environmental stability [4], and high thermal and electrical conductivity [5]. These properties make CNT very attractive for many structural applications, such as aerospace structures, body armor, and sporting goods [6]. However, methods that take full advantage of the excellent mechanical properties of microscale CNT remain elusive. Early studies of CNT-reinforced nanocomposites showed that CNT was an effective reinforcing phase for enhancing the mechanical properties of polymer matrices [7], but the extent of reinforcement was limited by the quality of dispersion, CNT alignment, and load-transfer efficiency between the CNT and the matrix. Thus, it seems that the full potential of CNTs have not yet been utilized in CNT assemblies like fibers, threads, and composites.

CNTs are chemically stable to large variety of environments; therefore, a poor interaction between the CNTs and their matrix is common in most applications where CNTs are used as reinforcing additives. Reactive sites known as functional groups need to be incorporated in order to improve 
their interaction with the medium. There are multiple CNT functionalization methods, most of them are chemical [8-11]. A very effective method is plasma functionalization, which modifies the surface morphology of CNTs by introducing defects and functional groups in the CNT surface [12]. Plasma has been used extensively for surface modification of multiple nanomaterials, and it has proven to be quite effective to improve dispersions and breaking up nanoparticle agglomerations [13-19]. Utilizing plasma methods to treat or modify carbon nanomaterials can widen the range of compatible polymer matrices. Tailoring CNT functional groups it is possible to increase their dispersion homogeneity within polymers, enhance the interfacial bonding to the polymer matrix, and achieve the desired properties of the resulting polymer nanocomposites [13].

Composite materials based on epoxy resins are widely used for applications where a high modulus, thermal stability, and solvent resistance are required [20]. Glycidyl amine epoxy resins are used as low temperature curing structural epoxy composite materials [21]. 4,4-methylenebis(N,N-diglycidylaniline) (MBDGA), a kind of glycidyl amine epoxy resin studied in this report, has four glycidyl epoxy groups in a molecular structure. Hypothetically, that means a molecule of MBDGA can react with four amine groups cross-linking one or more functional groups in the CNTs. After the reaction is complete, we expect that the tensile strength of CNT yarn functionalized with glycidyl amine resin will be much higher than pristine CNT thread. Although the cross-linking approach may be applicable to all CNTs, this manuscript is focused on MWCNTs.

CNT fibers and threads, terms used indistinctly in the literature, are forms of CNT assemblies that have shown performances below individual CNTs. Regardless of their assembly method, individual CNTs within the fiber are held together by van der Waals forces only. Covalent bond formation between CNTs is an approach that have potential to increase the mechanical strength of the assembly [22,23], as well as improve other physical properties [24]. Multiple cross-linking chemistries have been attempted but few of them have been focused on the mechanical strength improvements [22,23,25-27]; therefore, the search for an ideal reaction that will crosslink CNTs within the fiber continues. Here, we report a six-fold increase of the mechanical strength of the CNT threads after covalent cross-linking. Our strategy is to use free radical and ionic species in helium/nitrogen plasma in order to create active sites on CNT surface for bonding with ethylenediamine (EDA) molecules, thus enabling amine functionalization of the nanotubes. These amine functional groups will reacts with an epoxy based cross linker known as MBDGA.

\section{Experimental}

\subsection{Sample Preparation}

MWCNTs with outer diameters ranging 6-20 nm, wall number 2-10, and length 400-500 $\mu \mathrm{m}$ were manufactured from Nanoworld Laboratories of University of Cincinnati by a water-assisted CVD method [28-31]. MWCNT threads were assembled from drawable MWCNT arrays using the dry spinning method which starts with the synthesis of vertically-aligned drawable MWCNTs, typically between 400 and $500 \mu \mathrm{m}$ in length. The process starts with catalyst thin film deposition of Fe/Co alloys $\left(1.2 \mathrm{~nm}\right.$ ) on 4 -inch $\mathrm{Si}$ wafers that has $5 \mathrm{~nm} \mathrm{Al} \mathrm{O}_{3}$ layers deposited by sputtering (Kurt J. Lesker, Jefferson Hills, AL, USA) as a buffer layer. The Si wafer substrates were scribed and broken into 2-inch long and variable width (up to $1.5 \mathrm{inch}$ ) substrates that were loaded into a commercial CVD reactor ET3000 from CVD Equipment Corporation (Central Islip, NY, USA). The pressure during the growth process is 740 Torr. The reactor is heated to $400{ }^{\circ} \mathrm{C}$ for annealing under Ar, after 2 min the reactor is ramped to $750{ }^{\circ} \mathrm{C}$, and a $300 \mathrm{sccm}$ mixture of $\mathrm{C}_{2} \mathrm{H}_{4}$ and $1000 \mathrm{sccm}$ of Ar are introduced at this temperature for $20 \mathrm{~min}$. Upon growth completion, $30 \mathrm{sccm}$ of $\mathrm{H}_{2} \mathrm{O}$ and $2000 \mathrm{sccm}$ of Ar were delivered during cooling to promote CNT array detachment.

Drawable MWCNT arrays are ideal to make different diameter of MWCNT threads via simultaneous twisting and winding. A Surfx Atomflo 400D plasma system was used to create atmospheric pressure plasma from a mixture of helium and nitrogen gases $(30: 0.4 \mathrm{~L} / \mathrm{min})$ operating at 
$80 \mathrm{~W}$ (gases are ultra-high purity, from Wright Brothers, Montgomery, AL, USA). EDA in vapor form was injected into the plasma jet via flow of helium a t $0.5 \mathrm{~L} / \mathrm{min}$ through liquid EDA.

After toluene densification, MWCNT thread was functionalized with EDA, and pristine thread was kept unfunctionalized as a control. The functionalized MWCNT thread was then added to a $5 \mathrm{wt} \%$ MBDGA (Sigma-Aldrich, St. Louis, MO, USA) toluene solution and allowed to react at room temperature for $12 \mathrm{~h}$. After the cross-linking reaction, the thread was washed with toluene to remove the polymer excess at its surface. The mass of liquid curing agent (LCA), EDA, can be calculated by using the follow equation:

$$
M_{L C A}=\frac{M \times G}{H_{n}}
$$

- $M_{L C A}$-mass of liquid curing agent;

- $\quad M$-molecular weight of LCA;

- $G$-epoxide number of glycidyl amine epoxy;

- $H_{n}$-number of active hydrogen.

The EDA/MBDGA cross-linked thread was dried in an oven at $120^{\circ} \mathrm{C}$ for $30 \mathrm{~min}$ to vaporize the solvent.

\subsection{Characterization}

The surface morphology and diameter of CNT thread were observed under a scanning electron microscope (ESEM, Philips XL-30 Field emission, Eindhoven, The Netherlands). CNT thread samples were analyzed by Raman, in a Via micro-Raman spectrometer (Raman, Gluocestershire, UK), X-ray photoelectron spectroscopy (XPS, ESCALAB PHI-5300, Waltham, MA, USA) and Thermogravimetric Analysis and Differential Scanning Calorimeter (TA-DSC, NETZSCH STA-409PC, Burlington, MA, USA) to determine the structural characteristics and chemical elements of the material before and after EDA plasma-functionalization, as well as of MBDGA cross-linked CNT. The diameter of each CNT thread was obtained by observing twenty positions under SEM. When testing the mechanical properties of all thread samples, the active gauge length was $16 \mathrm{~mm}$ and a strain rate was of $1.0 \mathrm{~mm} \cdot \mathrm{min}^{-1}$, at least ten specimens were taken from the same thread and measured to obtain the average tensile strength of the yarn. The tensile strength-extension curves were recorded by an INSTRON 5948 Micro Tester equipped (Norwood, LA, USA) with a $5 \mathrm{~N}$ load cell. Thermogravimetric analysis took place under air flow at a rate of $30 \mathrm{~mL} \cdot \mathrm{min}^{-1}$ and heating rate of $5^{\circ} \mathrm{C} \cdot \mathrm{min}^{-1}$.

\section{Results and Discussion}

\subsection{Mechanical Properties and Chemical Reaction Mechanism}

CNTs within a dry spun thread are held together by simple van der Waals bonding only. Slipping between CNTs is the most predominant form of failure. Covalent bonding, a much stronger type of chemical bonding than van der Waals, is highly desirable and has already shown improvements of the thread strength by bridging CNTs within the thread. However, to make meaningful improvements in strength, the number of covalent bonds within the thread has to increase which can bring significant challenges. One of these challenges is the ability to create a high number of functional groups and effectively introduce the cross linker with the thread. Due to the large number of CNTs within a thread, $\left(10^{6}\right.$, approximately) [32] and the initial bundle density, effective penetration of the thread by the cross-linker will be limited unless thread diameter is significantly reduced. A proof of this hypothesis is supported by thread strength dependence from the diameter among the cross-linked threads. For diameters above $10 \mu \mathrm{m}$, the tensile strength of the EDA/MBDGA cross-linked threads decreased with increasing CNT thread diameter as shown Figure 1. We speculate that this is due to the number of amine groups we create by EDA plasma within the thread, and the cross-linking agent penetration depth in the thread. Plasma is an effective surface functionalization technique, however plasma functionalization may decay radially in a cylindrical object such as the CNT thread. Thus, 
the number of functional groups within the thread will decay from surface to the center; therefore, a more effective functionalization may be accomplished on small diameter CNT threads. The solvent (cross-linker) penetration should also be more effective when infiltrating small diameter CNT thread. Figure 2 shows the tensile stress-strain curves of pristine CNT thread and EDA/MBDGA cross-linked CNT threads. The starting threads named as "pristine thread" have an average tensile strength of $194 \mathrm{MPa}$ and extension at failure of about 5\%. After treatment with toluene, functionalized with EDA plasma, and cross-linked with MBDGA the tensile strength of MBDGA cross-linked CNT yarn increases to $1192 \mathrm{MPa}$ and extension at failure of $4.2 \%$.

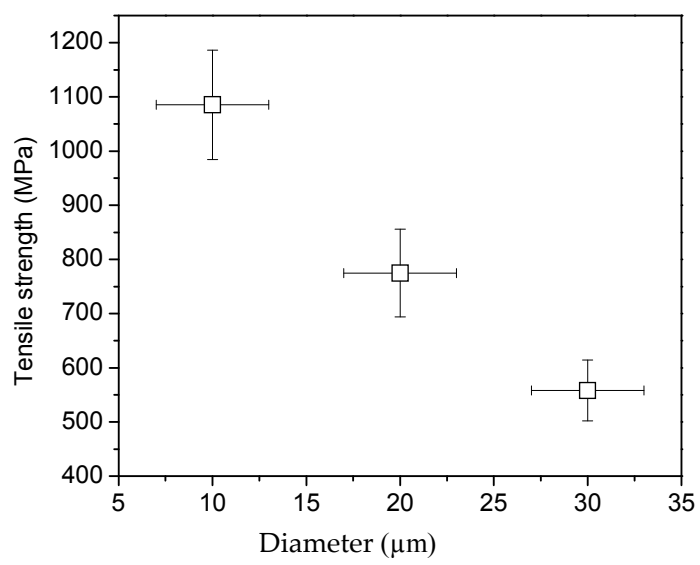

Figure 1. Tensile Strength of different diameter EDA/MBDGA cross-linked MWCNT threads.

The tensile strength of MBDGA cross-linked CNT thread shows a drastic improvement when compared to pristine CNT thread, as shown Figure 2. These results support the idea of covalent bond formation between CNTs within the thread, because neither the polymer itself nor the solvent densification could achieve such improvement alone. To understand and explain our results, we propose a chemical reaction mechanism shown in Figure 3. As shown, CNTs are functionalized by reactive chemical amine species generated by the plasma; therefore, it is reasonable to think that EDA plasma will generate two types of functional groups: with one and two $\mathrm{N}$ in the reactive amine group site. These intermediate products will react with one of the epoxy groups on the MBDGA following the reaction paths: A and B (Figure 3). We think the cross-linking effectiveness of MBDGA is high because each of the byproducts generated in step A and B, have a potential to react with additional three amines groups in the same or neighboring CNTs. Ideally, the MBDGA should bridge up to four CNTs within the tread if the arrangement and position allows.

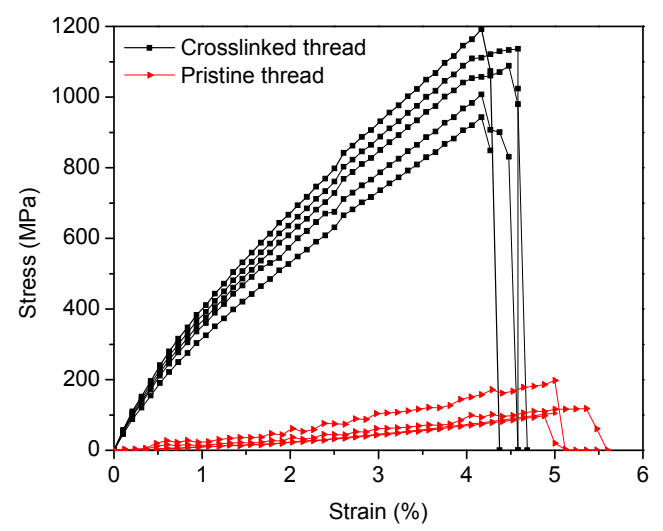

Figure 2. Tensile strength-extension curve of pristine MWCNT thread compared to EDA/MBDGA cross-linked MWCNT thread. 


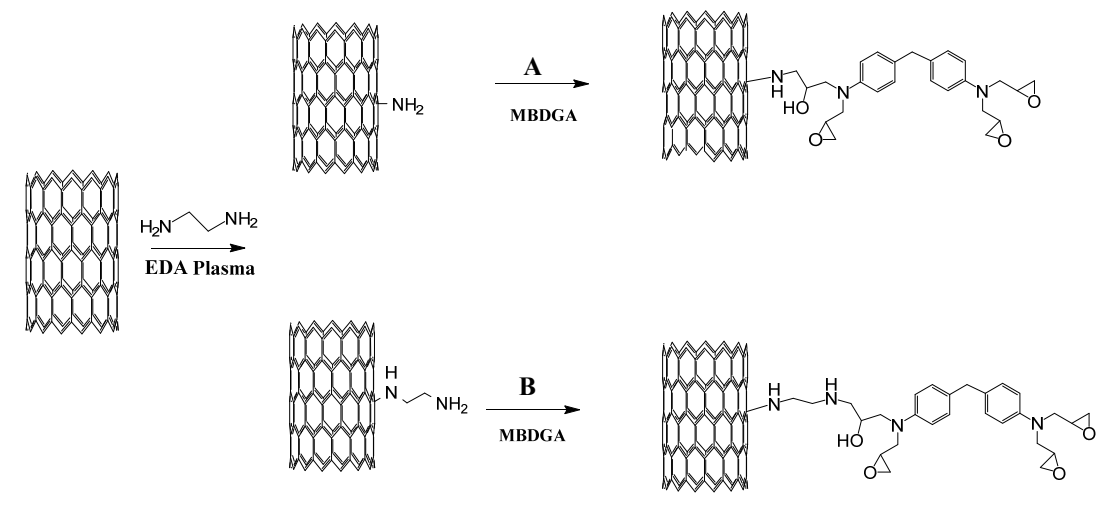

Figure 3. Proposed cross-linking reaction mechanism of EDA functionalized MWCNTs in threads and fibers. Epoxy functional groups in MBDGA are the actives sites that promote covalent bond formation between MWCNTs.

\subsection{The Effects of Defects and Functional Groups}

\subsubsection{SEM Analysis}

An encouraging fact is that CNT thread after covalent cross-linking shows a smaller diameter than its pristine counterpart, suggesting that density of CNTs increased within the thread by reducing the distance between them. SEM images shown in Figure 4a,b support this observation. Typically pristine CNT threads from drawable CNT arrays have low density, which increase as they are compressed or exposed to organic solvents. The threads are assembled through simultaneous twisting and drawing CNT bundles from vertically-aligned MWCNTs, they obtain their cylindrical shape due to the continuous twisting. The tested thread samples in our studies had a uniform diameter of $13 \mu \mathrm{m}$, as shown in Figure 4a. After EDA plasma-functionalization, MBDGA cross-linking, and toluene washing the thread retains a uniform diameter, though the diameter was reduced to $9 \mu \mathrm{m}$, as shown in Figure $4 \mathrm{~b}$.

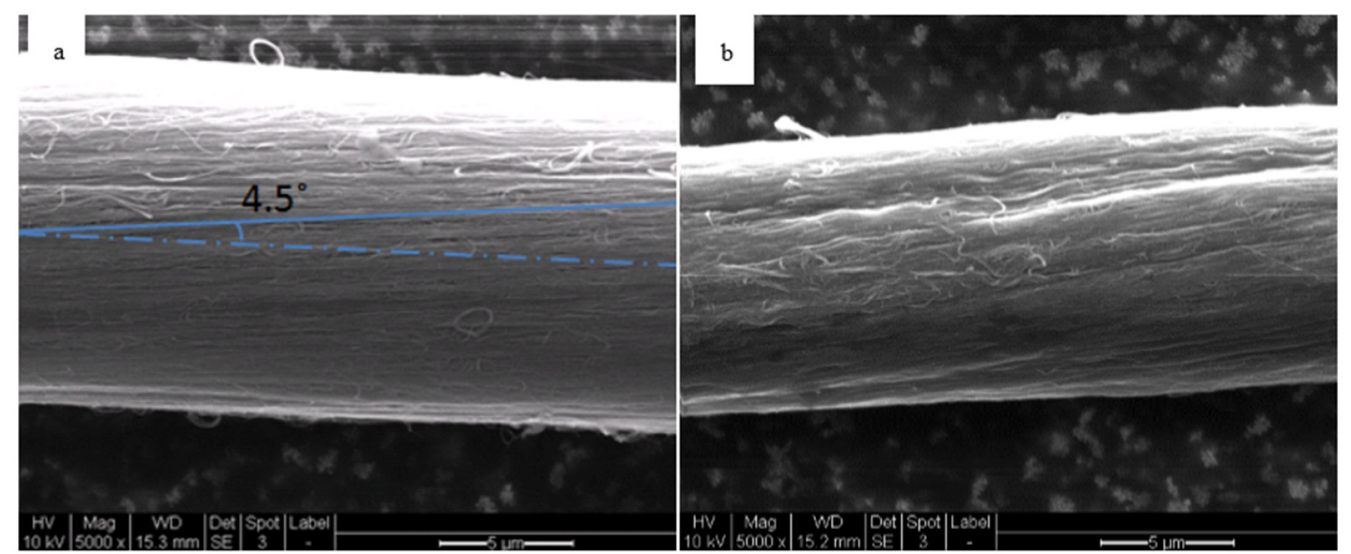

Figure 4. SEM images of (a) pristine; and (b) EDA/MBDGA cross-linked MWCNT.

\subsubsection{Raman Analysis}

The continuous generation of highly reactive species generated in the plasma environment, and subsequent collision with MWCNTs is a simple approach to chemical functionalization. Plasma ions are known for their ability to modify surface chemistry of materials which is supported by its Raman spectra $\left(514 \mathrm{~nm}\right.$ ) that shows an increase of $I_{\mathrm{D}} / I_{\mathrm{G}}$ ratios (from 0.69 to 1.68) for functionalized MWCNTs compared to their pristine counterparts, Figure 5a. Main peaks D, G, and D', as well as the Raman 
peaks shifting after functionalization are visualized. Furthermore, $\mathrm{D}^{\prime}$ peak generally associated with the disorder on CNTs shows a relative intensity increase; $\mathrm{D}^{\prime}$ that is barely visible in the pristine MWCNTs becomes a quite evident peak after functionalization. Figure $5 b$ shows the positions of the peaks and their correspondent Raman shifts of the main peaks. As much as $10 \mathrm{~cm}^{-1} \mathrm{shifting}$ in the $G$ peak is observed for amine-functionalized compared to pristine MWCNTs. Figure 5b also suggests that current plasma treatment does produce moderate damage to the CNTs, because similar Raman spectra have been reported for plasma-functionalized and pristine CNTs [33]. Fortunately this defects introduced by plasma treatment into CNT surface, are reactive sites that are used for replacing by amine functional groups. To increase the amount of amine groups, nitrogen plasma is used to functionalize CNTs.
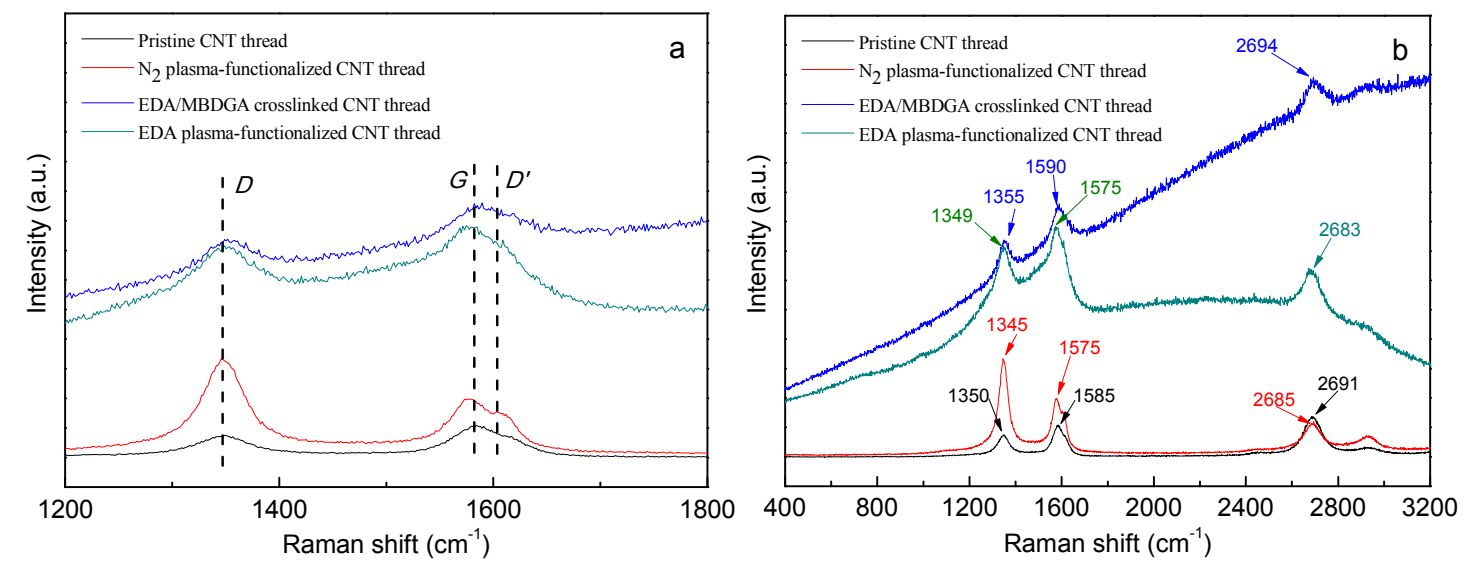

Figure 5. Raman spectra (514 nm) of pristine, plasma functionalized, EDA/MBDGA cross-linked MWCNTs threads; (a) shows Raman shifts of main peaks D, G, and D' at pristine state and after functionalization of MWCNTs; (b) larger Raman shift window visualizing Raman shifts for each peak and the shift of $\mathrm{G}$ peak after functionalization.

\subsubsection{XPS Analysis}

Additional evidence of functionalization is provided by X-ray Photoelectron Spectroscopy (XPS). Surface element composition of functionalized CNTs are analyzed and compared to XPS of pristine CNTs. In the XPS spectra, each peak is fitted by using the binding energy of standard carbon, $284.5 \mathrm{eV}$ [34]. Figure 6a shows and XPS elemental survey of pristine CNTs which shows the presence of elemental carbon only. Figure $6 \mathrm{~b}$ shows the main peak position at $284.5 \mathrm{eV}$ is for pure $s p^{2}$ hybridized CNTs and the peak position at $290.5 \mathrm{eV}$ is for characteristic shakeup line of carbon in aromatic compounds ( $\pi-\pi^{*}$ transition) [34,35]. After functionalization with EDA plasma, the nitrogen amount on the CNT surface reaches $17.4 \%$ as shown in the XPS survey Figure 6c. The presence of N suggests EDA functionalization of the CNTs at the CNT thread surface. The asymmetric peak at $284.5 \mathrm{eV}$ is assigned to $s p^{2}$-hybridized graphitic carbon atoms and $s p^{2}$ carbon atoms bound to hydrogen. Figure $6 \mathrm{~d}$ shows C1s region of the XPS spectra on EDA plasma functionalized CNTs. In Figure 6d shows the carbon nitrogen $(\mathrm{C}-\mathrm{N})$ bond peak at $286.73 \mathrm{eV}$ and carbon oxygen $(\mathrm{C}-\mathrm{O})$ bond peak at $288.25 \mathrm{eV}$. $\mathrm{C}-\mathrm{N}$ bond can be observed after EDA plasma functionalization. The peak at $288.4 \mathrm{eV}$ in Figure $6 \mathrm{~d}$, is a typical peak of carbon atoms bounded to oxygen atoms by single bonds caused by high temperature oxidation of CNTs. In Figure 6d, the peak at $285.59 \mathrm{eV}$ originates from $s p^{3}$-hybridized carbon atoms which also shows that defect are introduced onto the CNT surface [35,36]. The peak at $399.72 \mathrm{eV}$ in Figure 6e comes from the amines group on EDA plasma-functionalized CNTs, while the peaks appearing at $400.62 \mathrm{eV}$ and $401.28 \mathrm{eV}$ are from the $\mathrm{N}-\mathrm{C}$ bond and $\mathrm{NH}_{4}{ }^{+}$ion on EDA plasma-functionalized CNTs $[35,36]$. The peaks in Figure $6 \mathrm{f}$ appearing at $532.01 \mathrm{eV}$ and $533.49 \mathrm{eV}$ represent $\mathrm{C}-\mathrm{O}$ and $\mathrm{C}=\mathrm{O}$ respectively from high temperature plasma oxidation [37]. The oxygen amount on the CNT surface reaches $8.1 \%$, shown in Figure 6c, after functionalized with EDA plasma. 

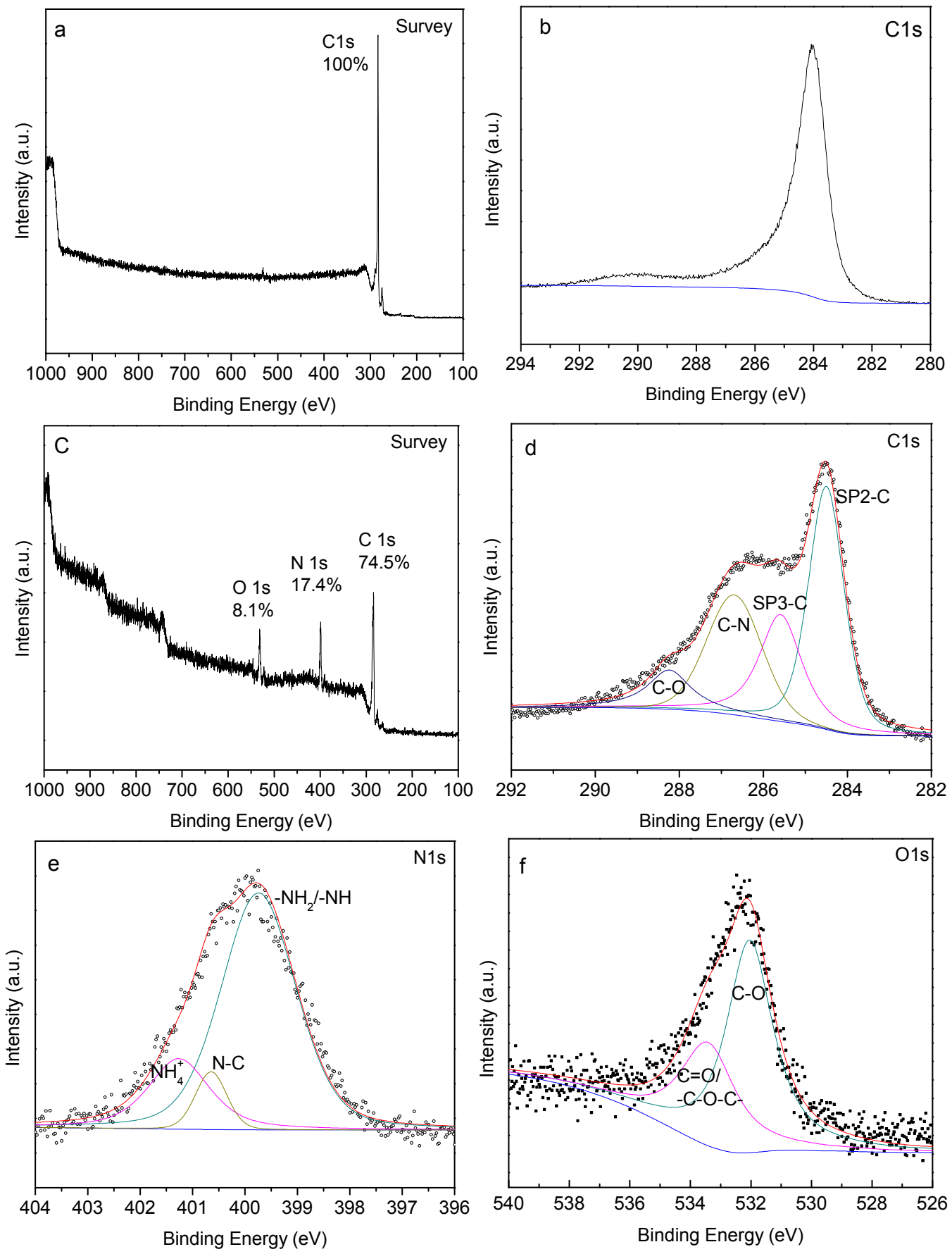

Figure 6. $\mathrm{XP}$ spectra of MWCNTs, (a) pristine survey; (b) pristine $\mathrm{C} 1 \mathrm{~s}$ region and EDA plasma-functionalized CNT; (c) survey; (d) C1s region; (e) N1s region; and (f) O1s region.

XPS of EDA/MBDGA cross-linked CNTs are shown in Figure 7. A Comparison of nitrogen and oxygen mass percentage of EDA plasma-functionalized CNTs (Figure 6c) and the EDA/MBDGA cross-linked CNTs (Figure 7a) shows that nitrogen mass decreasing from $17.4 \%$ to $2.7 \%$, and the oxygen mass increase from $8.1 \%$ to $21.4 \%$. This increase in mass percentage of $\mathrm{N}$ and $\mathrm{O}$ on the XPS spectrum suggests that amine groups in the EDA plasma functionalized have reacted with epoxy groups present in MBDGA. The C1s region XPS spectra (Figure $7 \mathrm{~b}$ ) shows the peak position at $284.5 \mathrm{eV}$ is for $s p^{2}$ hybridized CNTs, the $\mathrm{C}-\mathrm{N}$ bond peak at $285.47 \mathrm{eV}$ and the $\mathrm{C}-\mathrm{O}$ bond peak at $288.36 \mathrm{eV}$. The small shift of the peak position compared with EDA plasma-functionalized CNTs (Figure 6d) can support the 
cross-linking reaction. In Figure 7c, the peak at $398.94 \mathrm{eV}$ mainly comes from the trialkylamine group that is formed after the reaction of amine groups on EDA plasma-functionalized CNT with epoxy groups present in MBDGA [36-38]. The peaks in Figure 7d appear at $530.50 \mathrm{eV}$ and $533.45 \mathrm{eV}$ are from the $\mathrm{C}-\mathrm{OH}$ for open-ring reaction of epoxy groups present in the cross-linker with amine groups of the EDA plasma-functionalized CNTs and the $\mathrm{C}-\mathrm{O}-\mathrm{C}$ for open-ring reaction of epoxy groups in MBDGA with another epoxy group respectively during the cross-linking reaction [36]. A peak at $528.80 \mathrm{eV}$ comes from free oxygen on the surface of EDA/MBDGA cross-linked CNT. The XPS spectra are consistent with the chemical reaction mechanism shown in Figure 3. The robust covalent bonds formed between the amino groups of functionalized MWNTs and epoxy matrix can provide stronger interfacial shear stress, thereby improving the mechanical properties of the epoxy matrix [39].
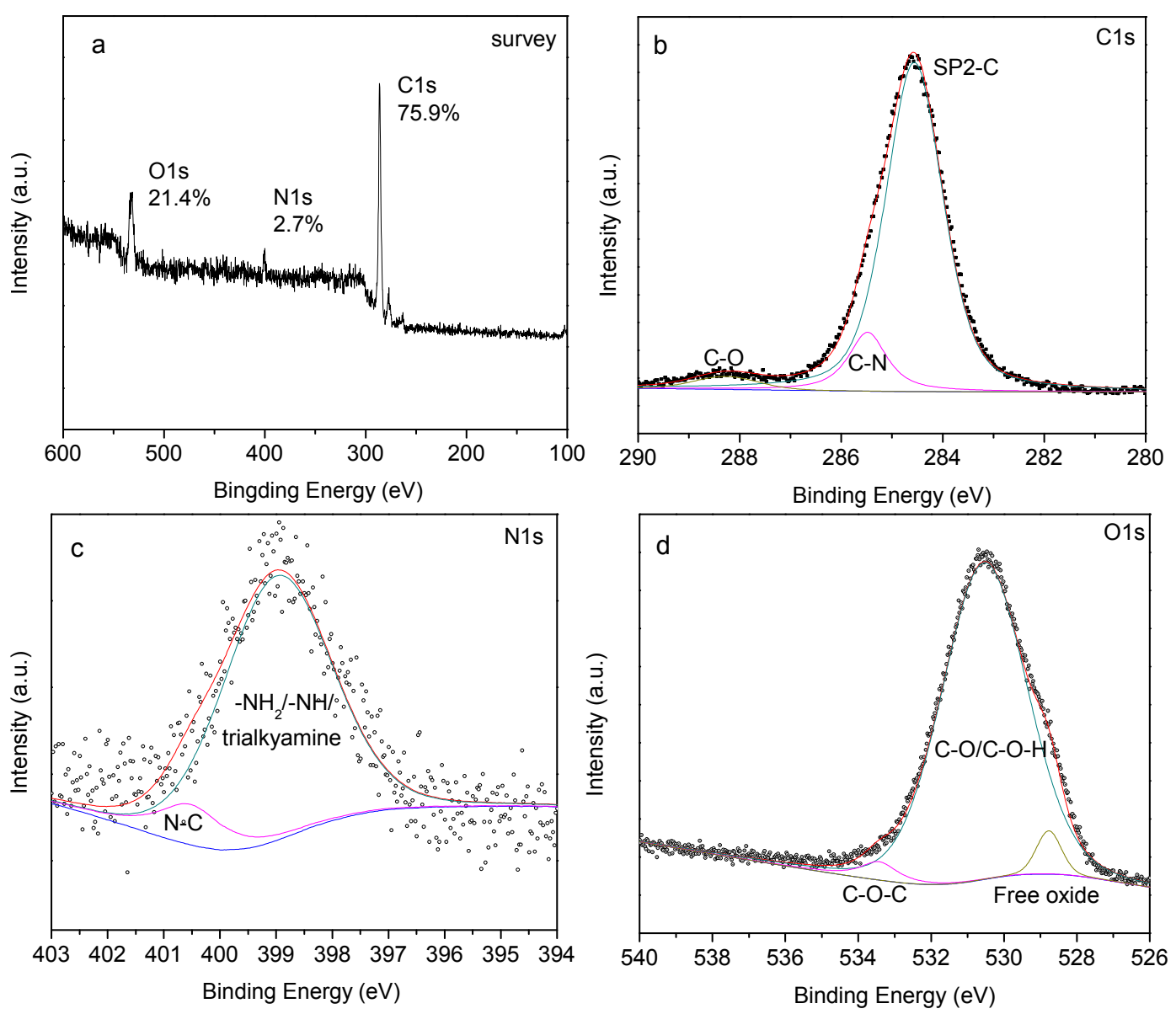

Figure 7. XP spectra of EDA/MBDGA cross-linked MWCNTs (a) survey; (b) C1s region; (c) N1s region; and (d) O1s region.

\subsubsection{Thermogravimetric Analysis}

TGA is carried out in air for the CNTs samples from $50{ }^{\circ} \mathrm{C}$ to $750{ }^{\circ} \mathrm{C}$ (Figure 8). Pristine CNTs remain fairly stable up to $500{ }^{\circ} \mathrm{C}$, after which they begin to oxidize rapidly. Peak oxidization occurs just below $700{ }^{\circ} \mathrm{C}$, and takes place across a single, well defined peak Figure 8a. The plasma-treated and cross-linked thread, in contrast, does not remain stable during heating; there is a steady mass loss from the onset of heating, which increases rapidly towards the end of the heating cycle. The mass loss at the outset is likely to be volatiles, perhaps residual solvent; this material is driven off by $200^{\circ} \mathrm{C}$. There are several cusps in the subsequent portions of the TGA trace, but the first derivative is unable to resolve them into clear peaks, Figure $8 \mathrm{~b}$. These are probably the polymer crosslinks decomposing but the limited polymer concentration may contribute to achieve well defined peaks. The last part of 
the TGA trace, starting around $500-550{ }^{\circ} \mathrm{C}$, shows a precipitous decline; the first derivative shows a clear peak at about $600^{\circ} \mathrm{C}$. This last region most likely corresponds to the CNT materials. Comparing the range of the first derivative peak with the mass loss in the thermogravimetric trace, it seems that CNTs constitute about $60 \%$ the material by mass. These CNTs have more defects than pristine material, as evidenced by the lower oxidation peak.

Another important property of CNT threads is their electrical conductivity. Comparison of conductivities between pristine and EDA/MBDGA cross-linked CNT threads are shown in Table 1. Electrical conductivity of pristine CNTs is $3984.26 \mathrm{~S} \cdot \mathrm{cm}^{-1}$ is higher than of cross-linked CNTs, $2123.40 \mathrm{~S} \cdot \mathrm{cm}^{-1}$. However, among the cross-linked MWCNTs it appear that larger diameter threads are more conducting than small diameter ones, suggesting that plasma functionalization may be more effective for small diameter threads. We think that further studies are needed to explain this reduction in conductivity after functionalization, although it seems reasonable to think that presence of plasma damaged CNTs as well as the presence of extra polymer within the thread will effectively reduce the electrical conductivity of the thread.

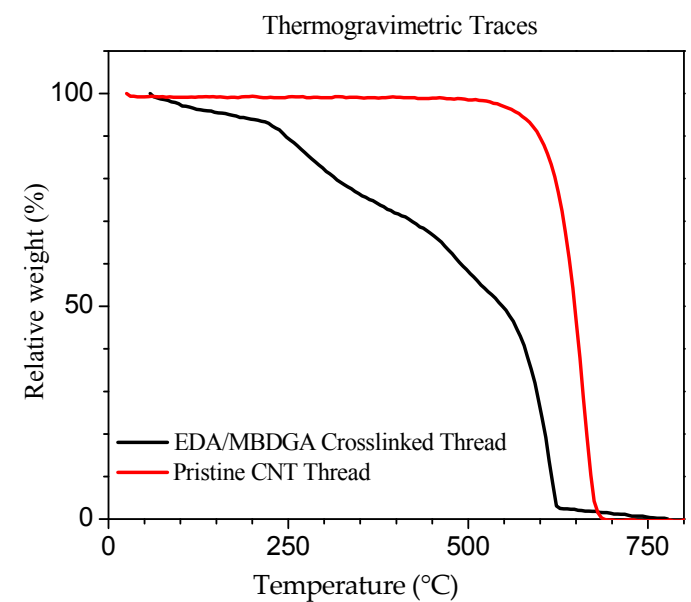

(a)

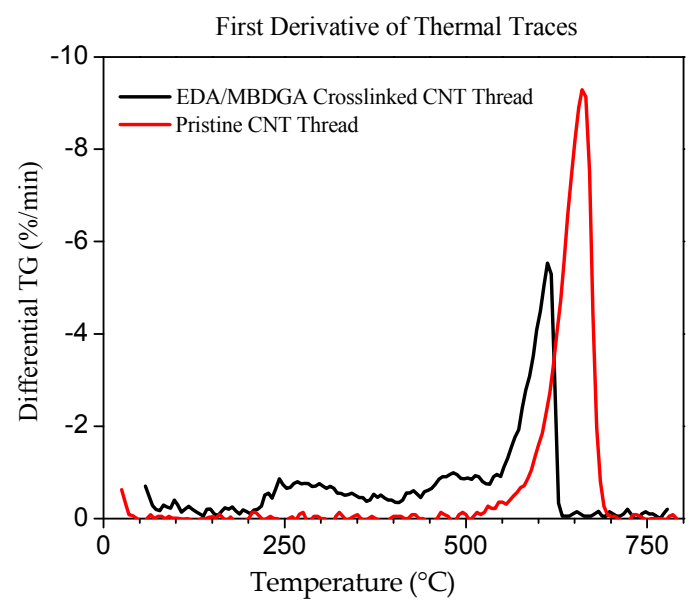

(b)

Figure 8. TGA curves of pristine MWCNT thread and EDA/MBDGA cross-linked MWCNT thread. (a)Thermogravimetric traces; (b) First derivative of thermal traces.

Table 1. Electrical conductivity of MWCNT threads.

\begin{tabular}{ccc}
\hline Sample & Conductivity $\left(\mathbf{S} \cdot \mathbf{~ c m}^{-1}\right)$ & Standard Deviation $\mathbf{( S \cdot \mathbf { c m } ^ { - 1 } )}$ \\
\hline Pristine CNT thread & 3984.26 & 110.67 \\
EDA/MBDGA cross-linked CNT thread & 2123.40 & 86.16 \\
\hline
\end{tabular}

\section{Conclusions}

Mechanical and electrical properties of pristine CNT thread and EDA/MBDGA cross-linked CNT thread has been explored. Significant mechanical strength improvement has been observed in the cross-linked CNT threads. Pristine CNT thread and EDA/MBDGA cross-linked CNT threads have a tensile strength of 194 and $1192 \mathrm{MPa}$ and electrical conductivities of 3984.26 and $2123.40 \mathrm{~S} \cdot \mathrm{cm}^{-1}$, respectively. Raman spectroscopy supports successful functionalization of CNTs on the threads. The conducted XPS study supports EDA plasma functionalization of the thread conforming CNTs. XPS also supports the cross-linking, and quantified the nitrogen amount on the surface of the thread, which reached $17.4 \%$ for EDA plasma-functionalized CNTs and 2.7\% for MBDGA cross-linked CNTs. The above results support that amine groups on the CNT surface reacted with epoxy group present in the cross linker MBDGA. TGA revealed that EDA/MBDGA cross-linked CNT thread has good thermal 
stability, though lower than CNTs. We think that the approach described in this work is a promising effort for bridging that gap between the extraordinary mechanical properties of individual CNTs and those of CNT assemblages such as threads and sheets.

Acknowledgments: We are grateful to the following Government agencies for their financial support: Chinese Jiangsu Overseas Research \& Training Program for University Prominent Young \& Middle-aged Teacher and President(2013-2014), Innovation Project of Chinese Jiangsu Province(2010-2013) and National Science Foundation through the following grants: CMMI-0727250; SNM-1120382; ERC-0812348.

Author Contributions: Qingyue Yu, Noe T. Alvarez, Vesselin Shanov, and Xinbao Zhu conceived and designed the experiments; Qingyue Yu performed the experiments; Qingyue Yu, Noe T. Alvarez, and Vesselin Shanov analyzed the data; Noe T. Alvarez, Peter Miller, Vesselin Shanov and Mark Schulz contributed materials; Qingyue Yu and Rachit Malik contributed reagents; Qingyue Yu, Noe T. Alvarez, Peter Miller, Rachit Malik, and Mark R. Haase contributed analysis tools; Qingyue Yu wrote the paper.

Conflicts of Interest: The authors declare no conflict of interest.

\section{References}

1. Dalton, A.B.; Collins, S.; Muñoz, E.; Razal, J.M.; Ebron, V.H.; Ferraris, J.P.; Coleman, J.N.; Kim, B.G.; Baughman, R.H. Super-tough carbon-nanotube fibers. Nature 2003, 423, 703. [CrossRef] [PubMed]

2. Demczyk, B.G.; Wang, Y.M.; Cumings, J.; Hetman, M.; Han, W.; Zettl, A.; Ritchie, R.O. Direct mechanical measurement of the tensile strength and elastic modulus of multiwalled carbon nanotubes. Mater. Sci. Eng. A 2002, 334, 173-178. [CrossRef]

3. Yu, M.F.; Siles, B.S.; Arepalli, S.; Ruoff, R.S. Tensile loading of ropes of single wall carbon nanotubes and their mechanical properties. Phys. Rev. Lett. 2000, 84, 5552-5555. [CrossRef] [PubMed]

4. Janas, D.; Vilatela, A.C.; Koziol, K.K.K. Performance of carbon nanotube wires in extreme conditions. Carbon 2013, 62, 438-446. [CrossRef]

5. McEuen, P.L.; Fuhrer, M.S.; Park, H. Single-walled carbon nanotube electronics. IEEE Trans. Nanotechnol. 2002, 1, 78-85. [CrossRef]

6. Zhang, X.; Li, Q.; Tu, Y.; Li, Y.; Coulter, J.Y.; Zheng, L.; Zhao, Y.; Jia, Q.; Peterson, D.E.; Zhu, Y. Strong carbon-nanotube fibers spun from long carbon-nanotube arrays. Small 2007, 3, 244-248. [CrossRef] [PubMed]

7. Gojny, F.H.; Wichmann, M.H.G.; Fiedler, B.; Schulte, K. Influence of different carbon nanotubes on the mechanical properties of epoxy matrix composites-A comparative study. Compos. Sci. Technol. 2005, 65, 2300-2313. [CrossRef]

8. Bahr, J.L.; Yang, J.; Kosynkin, D.V.; Bronikowski, M.J.; Smalley, R.E.; Tour, J.M. Functionalization of carbon nanotubes by electrochemical reduction of aryl diazonium salts: A bucky paper electrode. J. Am. Chem. Soc. 2001, 123, 6536-6542. [CrossRef] [PubMed]

9. Alvarez, N.T.; Li, Y.; Schmidt, H.K.; Tour, J.M. Selective redox chemistry with metallic swnt. 2008. unpublished.

10. Cai, J.Y.; Min, J.; McDonnell, J.; Church, J.S.; Easton, C.D.; Humphries, W.; Lucas, S.; Woodhead, A.L. An improved method for functionalisation of carbon nanotube spun yarns with aryldiazonium compounds. Carbon 2012, 50, 4655-4662. [CrossRef]

11. Kim, S.W.; Kim, T.; Kim, Y.S.; Choi, H.S.; Lim, H.J.; Yang, S.J.; Park, C.R. Surface modifications for the effective dispersion of carbon nanotubes in solvents and polymers. Carbon 2012, 50, 3-33. [CrossRef]

12. Banerjee, S.; Wong, S.S. Rational sidewall functionalization and purification of single-walled carbon nanotubes by solution-phase ozonolysis. J. Phys. Chem. B 2002, 106, 12144-12151. [CrossRef]

13. Ritts, A.C.; Yu, Q.; Li, H.; Lombardo, S.J.; Han, X.; Xia, Z.; Lian, J. Plasma treated multi-walled carbon nanotubes (MWCNTs) for epoxy nanocomposites. Polymers 2011, 3, 2142-2155. [CrossRef]

14. Gao, Y.; He, P.; Lian, J.; Wang, L.; Qian, D.; Zhao, J.; Wang, W.; Schulz, M.J.; Zhang, J.; Zhou, X.; et al. Improving the mechanical properties of polycarbonate nanocomposites with plasma-modified carbon nanofibers. J. Macromol. Sci. 2006, 45, 671-679. [CrossRef]

15. Shi, D.; Lian, J.; He, P.; Wang, L.M.; van Ooij, W.J.; Schulz, M.J.; Liu, Y.; Mast, D. Plasma deposition of ultrathin polymer film on carbon nanotubes. Appl. Phys. Lett. 2002, 81, 5216-5218. [CrossRef]

16. Yu, Q.; Kim, Y.J.; Ma, H. Plasma treatment of diamond nanoparticles for dispersion improvement in water. Appl. Phys. Lett. 2006, 88. [CrossRef] 
17. Avila-Orta, C.A.; Cruz-Delgado, V.J.; Neira-Velazquez, M.G.; Hernandez-Hernandez, E.; Mendez-Padilla, M.G.; Medellın-Rodriguez, F.J. Surface modification of carbon nanotubes with ethylene glycol plasma. Carbon 2009, 47, 1916-1921. [CrossRef]

18. Naseh, M.V.; Khodadadi, A.A.; Mortazavi, Y.; Pourfayaz, F.; Alizadeh, O.; Maghrebi, M. Fast and clean functionalization of carbon nanotubes by dielectric barrier discharge plasma in air compared to acid treatment. Carbon 2010, 48, 1369-1379. [CrossRef]

19. Wei, H.; Wei, Y.; Wu, Y.; Liu, L.; Fan, S.; Jiang, K. High-strength composite yarns derived from oxygen plasma modified super-aligned carbon nanotube arrays. Nano Res. 2013, 6, 208-215. [CrossRef]

20. Chen, X.; Wudl, F.; Mal, A.K.; Shen, H.; Nutt, S.R. New thermally remendable highly cross-linked polymeric materials. Macromolecules 2003, 36, 1802-1807. [CrossRef]

21. Rocks, J.; Rintoul, L.; Vohwinkel, F.; George, G. The kinetics and mechanism of cure of an amino-glycidyl epoxy resin by a co-anhydride as studied by FT-Raman spectroscopy. Polymer 2004, 45, 6799-6811. [CrossRef]

22. Kim, H.; Lee, J.; Park, B.; Sa, J.H.; Jung, A.; Kim, T.; Park, J.; Hwang, W.; Lee, K.H. Improving the tensile strength of carbon nanotube yarn via one-step double [2+1] cycloadditions. Korean J. Chem. Eng. 2015, 33. [CrossRef]

23. Min, J.; Cai, J.Y.; Sridhar, M.; Easton, C.D.; Gengenbach, T.R.; McDonnell, J.; Humphries, W.; Lucas, S. High performance carbon nanotube spun yarns from a crosslinked network. Carbon 2013, 52, 520-527. [CrossRef]

24. Kumar, R.; Rao, C.N.R. Assemblies of single-walled carbon nanotubes generated by covalent cross-linking with organic linkers. J. Mater. Chem. A 2015, 3, 6747-6750. [CrossRef]

25. Cheng, F.; Adronov, A. Suzuki coupling reactions for the surface functionalization of single-walled carbon nanotubes. Chem. Mater. 2006, 18, 5389-5391. [CrossRef]

26. Miller, S.G.; Williams, T.S.; Baker, J.S.; Solá, F.; Lebron-Colon, M.; McCorkle, L.S.; Wilmoth, N.G.; Gaier, J.; Chen, M.; Meador, M.A. Increased tensile strength of carbon nanotube yarns and sheets through chemical modification and electron beam irradiation. ACS Appl. Mater. Interfaces 2014, 6, 6120-6126. [CrossRef] [PubMed]

27. Leonard, A.D.; Hudson, J.L.; Fan, H.; Booker, R.; Simpson, L.J.; O'Neill, K.J.; Parilla, P.A.; Heben, M.J.; Pasquali, M.; Kittrell, C.; et al. Nanoengineered carbon scaffolds for hydrogen storage. J. Am. Chem. Soc. 2008, 131, 723-728. [CrossRef] [PubMed]

28. Alvarez, N.T.; Miller, P.; Haase, M.; Kienzle, N.; Zhang, L.; Schulz, M.; Shanov, V. Carbon nanotube assembly at near-industrial natural-fiber spinning rates. Carbon 2015, 86, 350-357. [CrossRef]

29. Jayasinghe, C.; Chakrabarti, S.; Schulz, M.J.; Shanov, V. Spinning yarn from long carbon nanotube arrays. J. Mater. Res. 2011, 26, 645-651. [CrossRef]

30. Alvarez, N.T.; Li, F.; Pint, C.L.; Mayo, J.T.; Fisher, E.Z.; Tour, J.M.; Colvin, V.L.; Hauge, R.H. Uniform large diameter carbon nanotubes in vertical arrays from premade near-monodisperse nanoparticles. Chem. Mater. 2011, 23, 3466-3475. [CrossRef]

31. Alvarez, N.T.; Orbaek, A.; Barron, A.R.; Tour, J.M.; Hauge, R.H. Dendrimer-assisted self-assembled monolayer of iron nanoparticles for vertical array carbon nanotube growth. ACS Appl. Mater. Interfaces 2010, 2, 15-18. [CrossRef] [PubMed]

32. Miao, M. Yarn spun from carbon nanotube forests: Production, structure, properties and applications. Particuology 2013, 11, 378-393. [CrossRef]

33. Gao, G.; Jin, Y.Z.; Kong, H.; Whitby, R.L.D.; Acquah, S.F.A.; Chen, G.Y.; Qian, H.; Hartschuh, A.; Silva, S.R.P.; Henley, S.; et al. Polyurea-functionalized multiwalled carbon nanotubes: Synthesis, morphology, and Raman spectroscopy. J. Phys. Chem. B 2005, 109, 11925-11932. [CrossRef] [PubMed]

34. Kim, J.A.; Seong, D.G.; Kang, T.J.; Youn, J.R. Effects of surface modification on rheological and mechanical properties of CNT/epoxy composites. Carbon 2006, 44, 1898-1905. [CrossRef]

35. Maldonado, S.; Morin, S.; Stevenson, K.J. Structure, composition, and chemical reactivity of carbon nanotubes by selective nitrogen doping. Carbon 2006, 44, 1429-1437. [CrossRef]

36. Lee, S.W.; Kim, B.S.; Chen, S.; Shao-Horn, Y.; Hammond, P.T. Layer-by-layer assembly of all carbon nanotube ultrathin films for electrochemical applications. J. Am. Chem. Soc. 2009, 131, 671-679. [CrossRef] [PubMed]

37. Kundu, S.; Wang, Y.; Xia, W.; Muhler, M. Thermal stability and reducibility of oxygen-containing functional groups on multiwalled carbon nanotube surfaces: A quantitative high-resolution XPS and TPD/TPR study. J. Phys. Chem. C 2008, 112, 16869-16878. [CrossRef] 
38. Kundu, S.; Xia, W.; Busser, W.; Becker, M.; Schmidt, D.A.; Havenith, M.; Muhler, M. The formation of nitrogen-containing functional groups on carbon nanotube surfaces: A quantitative XPS and TPD study. Phys. Chem. Chem. Phys. 2010, 12, 4351-4359. [CrossRef] [PubMed]

39. Shen, J.; Huang, W.; Wu, L.; Hu, Y.; Ye, M. Thermo-physical properties of epoxy nanocomposites reinforced with amino-functionalized multi-walled carbon nanotubes. Compos. Part A Appl. Sci. Manuf. 2007, 38, 1331-1336. [CrossRef] 